\title{
Mit neuem Online-Programm gegen kognitive Defizite
}

Die Häufigkeit kognitiver Störungen bei MS-Patienten steigt von rund $25 \%$ bei einem klinisch isolierten Syndrom über $40 \%$ bei schubförmiger MS auf mehr als $80 \%$ bei sekundär progredienter MS [Potagas C et al. J Neurol Sci 2008; 267: 100106] ( $\triangleright$ Abb. 1). Während hoch überlernte Routinehandlungen unverändert gut ausgeführt werden können, haben Betroffene vor allem in ungewohnten Situationen und beim gedanklichen Vorausplanen Probleme, erklärte PD Dr. Björn Tackenberg, geschäftsführender Oberarzt der Klinik für Neurologie der Philipps-Universität Marburg. Kognitiv beeinträchtigte MS-Patienten haben etwa ein erhöhtes Unfallrisiko im Straßenverkehr im Vergleich zu gesunden Personen oder kognitiv intakten MS-Patienten [Schultheis MT et al. Neurology 2001; 56: 1089-94].

Wie Dr. Eva-Maria Wicklein, Global Medical Affairs, Neurology, Bayer HealthCare, Berlin, ergänzte, können die kognitiven Defizite die Alltagsaktivitäten und insbesondere die Arbeitsfähigkeit der Patienten beeinträchtigen. Bayer HealthCare hat deshalb mit CogniFit ${ }^{\circledast}$ ein Trainingsprogramm entwickelt, das allen im BETAPLUS ${ }^{\circledast}$-Programm registrierten $\mathrm{Pa}$ tienten auf www.ms-gateway.de kostenlos zur Verfügung steht. Das computerbasier-

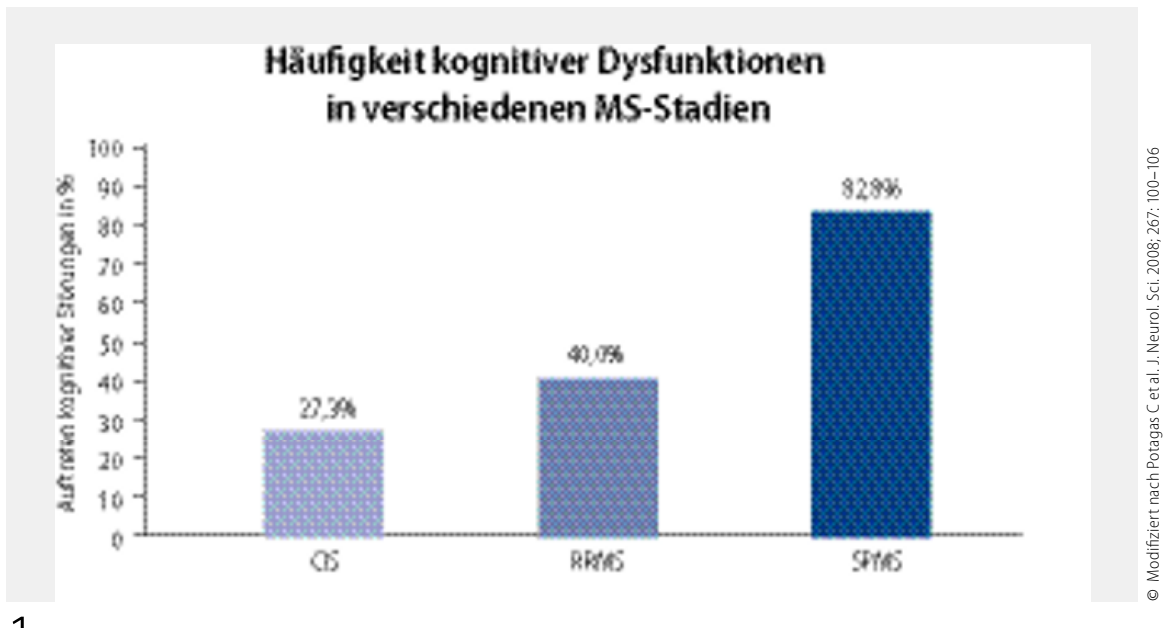

1 Häufigkeit kognitiver Störungen bei Patienten mit klinisch isoliertem Syndrom (CIS) schubförmiger MS (RRMS) und sekundär progredienter MS (SPMS) (nach Potagas et al. 2008)

te Online-Training bietet Übungen zu verschiedenen, gerade bei MS betroffenen kognitiven Domänen und gibt dem Patienten anschließend eine Übersicht über die von ihm erzielten Leistungen. In den nachfolgenden Sitzungen wird der Schwierigkeitsgrad den festgestellten Defiziten angepasst - mit Fokus auf die schwächeren Bereiche.

Nach Abschluss einer zwölfwöchigen Studie zeigten MS-Patienten, die das Training dreimal wöchentlich absolvier- ten, bessere Ergebnisse in den Bereichen Merkfähigkeit sowie Verarbeiten und Abrufen von Informationen als Patienten einer Kontrollgruppe [Shatil E et al. NeuroRehabilitation 2010; 26: 143 - 53].

Dr. Matthias Herrmann, freier Medizinjournalist

Meet the Expert "Kognition und MS -

Bayer denkt mit" beim 28. ECTRIMS,

Lyon, Frankreich, 12.10.2012

Veranstalter: Bayer HealthCare Deutschland

Major-Depression

\section{Die innere Uhr resynchronisieren}

Die Depression ist eine chronobiologische Erkrankung, die häufig mit einer gestörten 24-Stunden-Rhythmik assoziiert ist, erklärte Professor Göran Hajak, leitender Neurologe und Psychiater am Bamberger Klinikum am Michelsberg. Der Schlaf-Wach-Rhythmus sei bei depressiven Patienten häufig verflacht und phasenverschoben. Das Ausmaß der zirkadianen Dysregulation korreliere dabei mit dem Schweregrad der Depressivität.

Einen viel versprechenden neuen Ansatz in der Behandlung der Major-Depression sieht Hajak deshalb in Agomelatin $\left(\right.$ Valdoxan $\left.^{\circledast}\right)$. Der Melatoninagonist und $5-\mathrm{HT}_{2 \mathrm{C}}$-Antagonist zeigte in klinischen Studien neben der antidepressiven Wirksamkeit auch deutliche Verbesse- rungen der Schlafqualität bei Erhalt der Aktivität am Tage, ohne die Schlafarchitektur zu verändern.

Dass sich Agomelatin auch in der täglichen Praxis bewährt, belegte eine große deutsche nicht-interventionelle Studie mit mehr als 3.300 Patienten. Erste Daten einer Subgruppenanalyse, die Professor Gerd Laux, Psychiater aus Wasserburg, vorstellte, zeigen auch eine rasche und anhaltende Wirkung bei schwerer Depression und Altersdepression. Bereits in den ersten zwei Wochen besserten sich die Symptome auf der Montgomery-Asberg Depression Rating Scale (svMADRS) bei etwa jedem zweiten Teilnehmer um mindestens $20 \%$. Nach dreimonatiger Behandlung erreichten $48 \%$ bezie- hungsweise 59\% der beiden Gruppen eine Remission. Die meisten Ärzte bewerteten die Verträglichkeit von Agomelatin als sehr gut oder gut, Nebenwirkungen, die bei etwa $10 \%$ der Patienten auftraten, waren meist milde und unspezifisch. Wegen der Möglichkeit eines Transaminasenanstiegs sind Leberenzymkontrollen bei Therapiebeginn, nach drei, sechs, zwölf und 24 Wochen durchzuführen. Als positiv wertete Laux die Gewichtsneutralität der Substanz und den Erhalt der sexuellen Funktion.

Dr. Martina-Jasmin Utzt, freie Medizinjournalistin

Satellitensymposium „Depression: Eine komplexe systemische Erkrankung" beim DGPPNKongress, Berlin, 22.11.2012; Veranstalter: Servier 